\title{
Deformation mechanism of neutron irradiated Al-B based on SEM-DIC
}

\author{
Jiaqi Xiang ${ }^{1}$, Qijie Feng ${ }^{3}$, Junchao Cheng ${ }^{1}$, Lei Lu ${ }^{1}$, Junyu Huang ${ }^{2}$, and Zhengye Zhong, ${ }^{1}$ * \\ ${ }^{1}$ School of Materials Science and Engineering, Southwest Jiaotong University, Chengdu, Sichuan, \\ P.R. China \\ ${ }^{2}$ The Peac Institute of Multiscale Sciences, Chengdu, Sichuan, P. R. China \\ ${ }^{3}$ Institute of Nuclear Physics and Chemistry, Chinese Academy of Engineering Physics, Mianyang, \\ P.R. China
}

\begin{abstract}
The electron backscatter diffraction (EBSD) characterization of irradiated Al-B shows that there is a high concentration defect region around the borides. Nanoscale speckle particles were successfully prepared on the surface of Al-B before and after irradiation, and then the mesoscale strain during in-situ deformation was obtained by digital image correlation (DIC) technique. The results shows that slip band bypass such an area through cross slips with slip band deflection. Transmission electron microscopy (TEM) indicates that abundant helium bubbles exist in the deflected slip band area pinning the dislocations.
\end{abstract}

\section{Introduction}

Neutron radiation damage seriously affects the structure and mechanical properties of materials in service, threatens the safety of reactor operation and increases the operating cost [1]. However, due to the limitations of experimental conditions, the research on the effects of reactor neutron irradiation on the mechanical properties of materials is mainly focused on the molecular dynamics simulation calculation [2,3], and its applicability in the real irradiation environment has yet to be confirmed. At present, the experimental research in this field mainly focuses on the simulation of neutron irradiation by heavy ion irradiation, and the research scope is mostly limited to the nanoscale [4-6], and the application of experimental results at the macro scale is very limited. Therefore, it is urgent to carry out the research on the effect of neutron irradiation on metal deformation mechanism at the mesoscopic scale.

Digital image correlation (DIC) of scanning electron microscope (SEM) images is a powerful technique for measuring full-field deformation at mesoscopic scale [7]. A major challenge in applying this technology is to produce speckle patterns small enough to facilitate full-field measurements at high spatial resolution and high magnification $[8,9]$. In this paper, DIC is applied to investigate the mesoscale deformation of the irradiated aluminum matrix just around the borides based on scanning electron microscopy (SEM). The experimental results show that the slip bands will be seriously hindered, and its

${ }^{*}$ Corresponding author: zyzhong@pims.ac.cn 
deformation is uneven and discontinuous; the matrix around the boride particles is severely hardened which causing the slip band deflection near this area.

\section{Materials and experiments}

\subsection{Initial material characterization}

The experimental material in this paper is high purity aluminium (99.999 wt\% purity) containing $0.07 \mathrm{wt} \%{ }^{10} \mathrm{~B}$. The mixture is prepared via melting high purity aluminum with ${ }^{10} \mathrm{~B}$ particles in an arc furnace at 1200 for $30 \mathrm{~min}$. The melt is then poured into a $26 \mathrm{~mm}$ diameter cylinder and immediately cooled with liquid nitrogen, to prevent boron segregation and borides formation. The SEM and EDS surface scanning analysis (Fig.1) of Al-B show that the boride particles are distributed randomly in the matrix, which are in the shape of long strips, the length of which is about $20 \mu \mathrm{m}$, the width of which is about $1 \mu \mathrm{m}$.

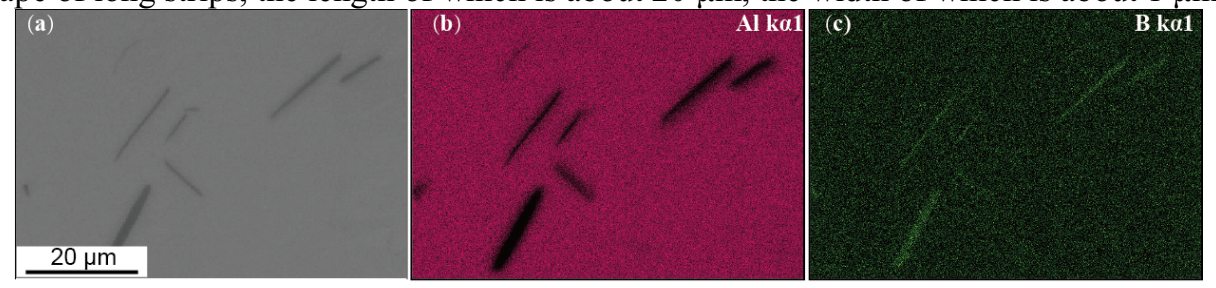

Fig. 1. Micromorphology and element distribution of Al-B.

\subsection{EBSD characterization before and after neutron irradiation}

The Al-B samples are irradiated to approximately $5.1 \times 10^{20} \mathrm{n} / \mathrm{cm}^{2}$ in China Mianyang Research Reactor, generating a helium concentration of $\sim 3.2 \times 10^{19} \mathrm{~cm}^{-3}$. In addition to usual neutron irradiation damage, mixing ${ }^{10} \mathrm{~B}$ into aluminum increases helium production through the transmutation reaction ${ }^{10} \mathrm{~B}(\mathrm{n}, \alpha){ }^{7} \mathrm{Li}$ under neutron irradiation, generating abundant helium in a relatively short time and forming large quantities of helium bubbles.

The Al-B samples before and after irradiation were characterized by EBSD, and the results are shown in the Fig. 2.

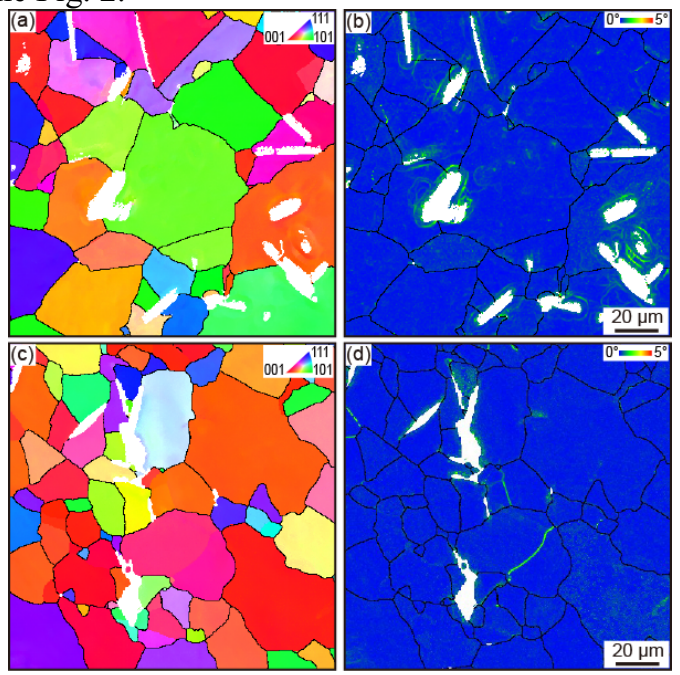

Fig. 2. EBSD characterization of the areas near boride particles. 
Fig. 2a-b are the IPF and KAM plots before irradiation, and Fig. 2c-d are the plots after irradiation, respectively. The areas where borides are shed after electrolysis sample preparation appear white (The electrolyte was $5 \mathrm{~mL} \mathrm{H}_{2} \mathrm{O}, 5 \mathrm{~mL} \mathrm{HClO}_{4}$, and $90 \mathrm{~mL}$ $\mathrm{C}_{2} \mathrm{H}_{5} \mathrm{OH}$. The electrolysis parameters were $20 \mathrm{~V}, 0.1 \mathrm{~A}, 120 \mathrm{~s}$ at $-40^{\circ} \mathrm{C}$ ). It was found that there was a ring region with significantly increased KAM value around the boride particles after irradiation.

\subsection{Nano speckle Preparation of nanoparticle speckle and in-situ deformation experiments}

After electrolytic polishing, the samples are coated with a $\sim 40$-nm-thick layer of silver, and immersed in a 1\% sodium chloride solution for 2 hours to form nano-speckles on the surface. A representative backscatter electron image (Fig. 3a) exhibits the large gray-scale contrast among silver particles, the aluminum matrix and a boron particle, given their different atomic masses. The silver particles (bright ones) are distributed homogeneously. The deposited silver particles have an average size of $\sim 70 \mathrm{~nm}$ (inset of Fig. 3a). The grayscale values of the SEM image are distributed in a wide range accordingly, where the mean intensity gradient is calculated as 53, and thus reliable DIC results can be obtained [4].
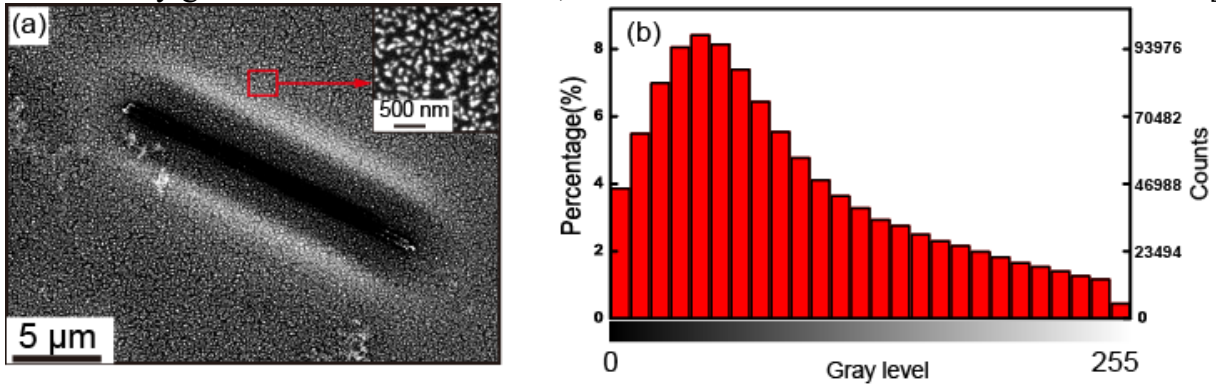

Fig. 3. Nano speckles and their gray level distribution.

Both the unirradiated and irradiated Al-B samples are subjected to in-situ uniaxial tension along with simultaneous SEM and EBSD characterization. The tensile tests are carried out in the SEM chamber with a homemade universal testing machine. The sample is a standard "dog bone" shape with gauge size of $4 \times 1 \times 1 \mathrm{~mm}$.

\section{Results and discussion}

\subsection{Comparison of tensile strain fields before and after neutron irradiation}

Fig. 4 presents the strain fields around borides particles (black areas) for the unirradiated and irradiated Al-B samples during tension. At a strain of $1 \%$, strain localization occurs and is manifested as a narrow high-strain band in both samples. The strain localization bands are induced by dislocation slip in aluminum, also known as slip bands. As the deformation proceeds, plastic strain accumulates further in the slip bands. Moreover, the slip bands in the unirradiated Al-B sample can approach the very edge of borides particles, and more slip bands can reach the particles at a higher strain level (Fig. 4a-c), indicating that the defects between the particles and the matrix which are induced by sample synthesis are not strong enough to stop slip bands from approaching the particles. While for the neutron irradiated Al-B sample, slip bands are not in contact with boron particles; in other words, the 
proximity to a boride particle is a "forbidden zone" for dislocations. Slip bands then have to bypass the boron particle through deflection (Fig. 4d-f).

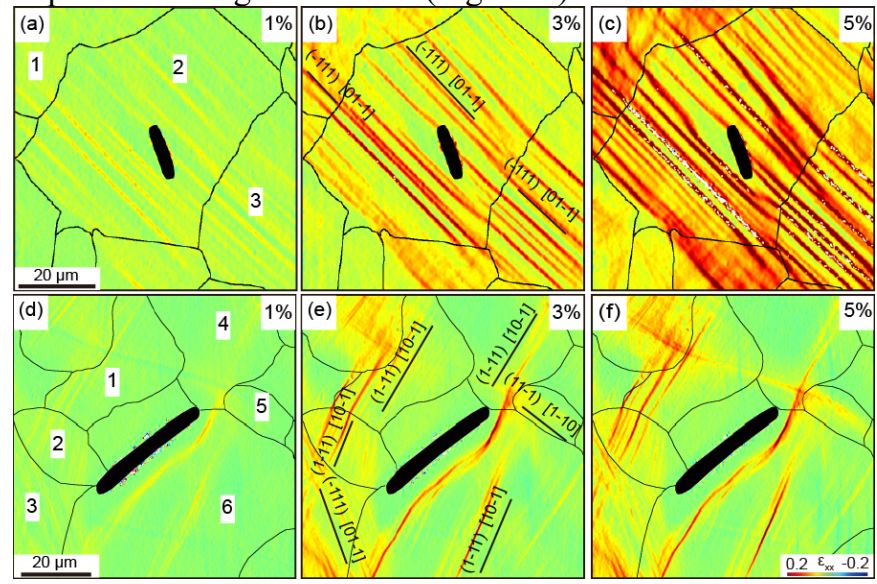

Fig. 4. EBSD characterization the areas near boride particles.

\subsection{TEM characterization results}

TEM analysis is performed at the proximity of a boride particle in the irradiated Al-B sample after tension (Fig. 5a), as marked by the red rectangle in Fig. 10a where a slip band deflects away from the particle. The cross-sectional view shows that underneath the slip band surface, a stripe with dense dislocations extends toward the interior of the sample (Fig. $5 b)$.
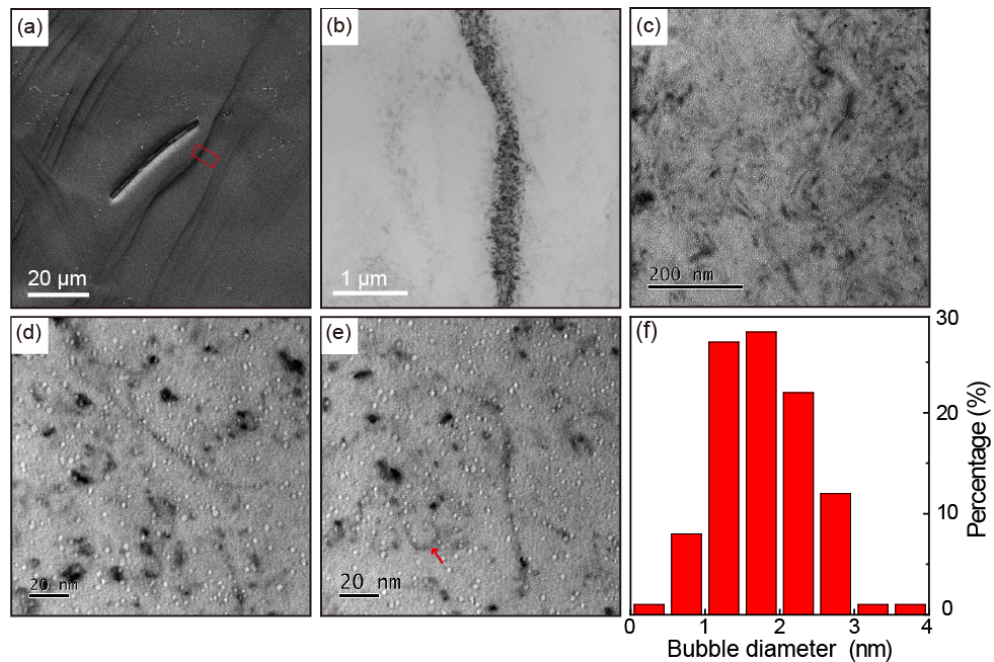

Fig. 5. TEM characterization for an irradiated sample after deformation. (a) SEM image of the deformed, neutron-irradiated Al-B sample. The red rectangle indicates the region for TEM sample preparation along the thickness direction via a focused ion beam. (b) TEM sample exposing the crosssection of the selected region in (a). (c-e) Enlarged views of different segments in the black band in (b). (f) Distribution of helium bubble size.

Three different regions in the stripe are magnified to examine the details inside (Fig. 5ce). Abundant helium bubbles, the white dots, distribute randomly in the stripe. The bubbles are practically spherical rather than faceted, indicating that the bubble interior is pressurized. 
According to the Young-Laplace equation, $\mathrm{P}_{\text {bubble }} \approx 4 \gamma_{\mathrm{s}} / \mathrm{d}$, where $\gamma_{\mathrm{s}}$ is the surface energy of $\mathrm{Al}\left(\sim 1.16 \mathrm{~J} / \mathrm{cm}^{2}\right)$ and $\mathrm{d}$ is the bubble diameter (the average bubble diameter $\sim 1.8 \mathrm{~nm}$, Fig. $5 \mathrm{f}$ ), the average pressure in the bubble is approximately $2.58 \mathrm{GPa}$. Moreover, dislocations are impeded by the randomly distributed helium bubbles, exhibiting curved blacklines. Dislocation loops are punched out by helium bubbles, as indicated by the arrow in Fig. 5e. The punched out interstitial dislocation loops may decompose into partials, resulting in an increase in the resistance of dislocation gliding through bubbles. Furthermore, the helium bubbles are active dislocation sources, generating dislocation loops once the pressure inside reaches a critical value.

Moreover, the increase in yield strength can be estimated with the dispersed barrier model for radiation induced small defect clusters serving as strong obstacles to dislocation motion [10], The dependence of yield strength increase $(\Delta \sigma)$ on defect cluster size and density can be described as

$$
\Delta \sigma=\mathrm{M} \alpha \mu \mathrm{b}(\mathrm{Nd})^{1 / 2}
$$

where $\mathrm{M}$ is the Taylor factor, $\alpha$ is the defect cluster barrier strength, $\mu$ is the shear modulus, $\mathrm{b}$ is the Burgers vector, $\mathrm{N}$ is the defect density, and $\mathrm{d}$ is the cluster diameter. The bubble density is estimated to be $\sim 1.7 \times 10^{23} \mathrm{~m}^{-3}$ from the TEM images in Fig. 5. (consistent with Ref. [11]), and thus $\Delta \sigma$ can reach as high as $\sim 360 \mathrm{MPa}$. It is relatively high, given that the yield strength of Al-B is below $100 \mathrm{MPa}$. The yield stress increase due to the presence of helium bubbles is reported around $500 \mathrm{MPa}$ at $300 \mathrm{~K}$ [12]. In the present study, $\alpha$ is set as 0.4 according to the reported barrier strength for visible defect clusters. $\alpha$ depends on helium bubble status, and can be better constrained with further modelling/theoretical efforts. Molecular dynamic simulation of an $\alpha$-iron shows that there is a monotonic increase in yield stress with increasing helium bubble diameter until the diameter reaches $\sim 2.3 \mathrm{~nm}$; after that bubble percolation occurs, resulting in yield stress decrease [13].

\section{References}

1. S. J. Zinkle, G. Was. Acta Mater, 61 (2013)

2. S. Hafez Haghighat, R. Schäublin. Philos. Mag, 90 (2010)

3. S. P. Yan, Y. Yu, H. Y. Wang, et al. Acta Phys.-Chim. Sin, 31 (2015)

4. M.S. Ding, L. Tian, W.Z. Han, et al. Phys. Rev. Lett, 117 (2016)

5. M.S. Ding, J.P. Du, L. Wan, et al. Nano Lett, 16 (2016)

6. H. Chen, Y. Cheng, C. Lin, et al,J. Nucl. Mater, 520 (2019)

7. Pan B, Qian K, Xie H, et al. Meas. Sci. Technol, 20 (2009)

8. Tong W ,Strain, 41 (2005)

9. C. Montgomery, B. Koohbor, N. R. Sottos.Exp. Mech, 59 (2019)

10. S. J. Zinkle, Y. Matsukawa, J. Nucl. Mater, 329 (2004)

11. N. M. Ghoniem, S. H. Tong, B. N. Singh, S. L. Z, Philos. Mag, 81 (2001)

12. E. Martinez, D. Schwen, A. Caro,Acta Mater 84 (2015)

13. N. N. Kumar, E. Martinez, B. K. Dutta, G. K. Dey, A. Caro, Phys. Rev. B, 87 (2013) 